\title{
3 Research Square

\section{Clinicopathological Characteristics and Endoscopic Features of Early Gastric Cancers Diagnosed After Helicobacter pylori Eradication: A Retrospective Study}

Hideki Ishibashi

Fukuoka University Faculty of Medicine https://orcid.org/0000-0003-3535-2906

Hidetoshi Takedatsu

Fukuoka University Faculty of Medicine

Taro Tanabe

Fukuoka University Faculty of Medicine

So Imakiire

Fukuoka University Faculty of Medicine Hiroki Matsuoka

Fukuoka University Faculty of Medicine

Hideki Yasuda

Fukuoka University Faculty of Medicine

Hideto Sakisaka

Fukuoka University Faculty of Medicine

Satoshi Matsuoka

Fukuoka University Faculty of Medicine

Yoshiyuki Kayashima

Fukuoka University Faculty of Medicine

Nobuaki Kuno

Fukuoka University Faculty of Medicine

Koichi Abe

Fukuoka University Faculty of Medicine

Sadahiro Funakoshi

Fukuoka University Faculty of Medicine

Fumihito Hirai ( $\square$ fuhirai@cis.fukuoka-u.ac.jp )

https://orcid.org/0000-0002-5493-5675

Research article 
Keywords: Early gastric cancer, Helicobacter pylori eradication, Endoscopic submucosal dissection, Endoscopic feature, Gastritis, Magnifying endoscopy

Posted Date: September 21st, 2020

DOI: https://doi.org/10.21203/rs.3.rs-36167/v2

License: (1) This work is licensed under a Creative Commons Attribution 4.0 International License. Read Full License 


\section{Abstract}

Background: Helicobacter pylori (H. pylori) infection is an important risk factor for developing gastric cancer. However, even after $H$. pylori eradication, early gastric cancer (EGC) can develop. We elucidated the characteristics of EGCs diagnosed after $H$. pylori eradication.

Methods: Thirty-six EGCs in 32 patients diagnosed after H. pylori eradication were defined as the eradication group (H. pylori-EG). The clinicopathological and endoscopic features were compared with those of 156 EGCs in 140 patients in the H. pylori-positive group (H. pylori-PG). Twenty-nine EGC lesions in the H. pylori-EG were further divided into two subgroups: the first included six lesions of no to mild atrophic mucosa around the EGC, and the second included 23 lesions of moderate to severe atrophic mucosa around the EGC. We compared them between the two subgroups.

Results: Endoscopic features of EGCs in the H. pylori-EG were characterized as small $(P=0.049)$ and of the depressed type $(P=0.022)$ compared with those in the H. pylori-PG. EGCs in the H. pylori-EG were detected in the upper region of the stomach more frequently than those in the H. pylori-PG $(P=0.002)$. Submucosal ECGs in the H. pylori-EG were more likely to be seen in the no to mild atrophic mucosa subgroup $(4 / 6,67 \%)$ compared with the moderate to severe atrophic gastric mucosa subgroup $(1 / 23,4 \%)$ $(P=0.003)$.

Conclusions: Careful follow-up endoscopies are necessary after H. pylori eradication.

\section{Background}

Infection with Helicobacter pylori (H. pylori) was classified as a gastric carcinogen by the World Health Organization in 1994 [1]. In patients with early gastric cancer (EGC) who underwent endoscopic resection, EGCs were detected in $\mathrm{H}$. pylori-positive patients at a significantly higher rate than in $\mathrm{H}$. pylorieradication patients [2]. In a multicenter randomized controlled trial in Japan, the incidence of metachronous cancer was 14.1 cases per 1,000 person-years in the $H$. pylori-eradication group and 40.5 cases per 1,000 personyears in the no-eradication group (hazard ratio $0.339,95 \%$ confidence interval $0.157-0.729, P=0.003$ ) [3]. The effectiveness of $H$. pylori eradication was proven by the decrease in the incidence of gastric cancer.

However, EGCs were sometimes detected after $H$. pylori eradication. Four previous studies showed the clinicopathological characteristics and endoscopic features of 239 EGCs after $H$. pylori eradication [4-7]. The median size of EGCs was $12.8 \mathrm{~mm}$ and the locations were upper (41 lesions, 17\%), middle (109 lesions, 44\%), and lower (94 lesions, 39\%) regions of the stomach. Endoscopic examination revealed that 34 were elevated lesions (18\%) and 152 (82\%) were depressed-type lesions [4-6]. Regarding depth of invasion, 206 (86\%) were intramucosal lesions and 33 (14\%) were submucosal invasive lesions [4-7]. EGCs after $H$. pylori eradication were histopathologically characterized as small and depressed lesions with low cell proliferation and gastric mucin phenotypes [4-8]. 
There are only a few reports concerning gastric cancer after $H$. pylori eradication, and therefore we conducted a retrospective study to investigate the clinicopathological characteristics and endoscopic features of EGCs diagnosed after $H$. pylori eradication.

\section{Methods}

\section{Study subjects}

The study was conducted at Fukuoka University from January 2009 to January 2018. The Medical Ethics Committee of Fukuoka University approved this study (IRB number: U20-01-011).

\section{Primary endpoint}

The primary endpoint of this study was to investigate the association between the clinicopathological characteristics and endoscopic features of EGCs diagnosed after H. pylori eradication. We enrolled 397 consecutive patients who underwent endoscopic submucosal dissection (ESD) of their EGC. Two hundred and twenty-five patients were excluded. The remaining 172 patients were divided into two groups. The $H$. pylori-eradication group (H. pylori-EG) comprised 36 EGCs in 32 patients who had undergone $H$. pylori eradication therapy $>1$ year before. The $H$. pylori-positive group (H. pylori-PG; control group) comprised 156 EGCs in 140 patients with active $H$. pylori infection. In both groups, EGC was treated by ESD (Fig. 1).

\section{Secondary endpoint}

It remains unclear whether the degree of mucosal atrophy affects the characteristics of EGC after eradication of $H$. pylori. Therefore, we compared the clinicopathological characteristics and endoscopic features between the two groups classified by the degree of gastric mucosal atrophy in the H. pylori-EG. We investigated the association between EGC characteristics and the degree of mucosal atrophy after $H$. pylori eradication. Twenty-nine EGC lesions, in which the degree of mucosal atrophy had been confirmed in ESD specimens, were divided into two subgroups according to endoscopic and histological examinations. These subgroups included the mild atrophic mucosa subgroup exhibiting no to mild atrophic mucosa around the EGC, and the moderate to severe atrophic mucosa subgroup displaying moderate to severe atrophic mucosa around the EGC (Fig. 1).

\section{Evaluation of $H$. pylori status}

Evaluation of $H$. pylori eradication treatment was based on the ${ }^{13} \mathrm{C}$-urea breath test (UBT) or serum immunoglobulin (Ig) G antibody test (E-plate, Eiken, Tokyo, Japan) and on histological assessment with Giemsa stain using endoscopic biopsy specimens. When both examinations were negative, we determined that $H$. pylori had been eradicated [4-6]. One hundred and forty patients in the control group were $H$. pylori-positive based on ${ }^{13} \mathrm{C}$-UBT or serum IgG antibody and on histological assessment with Giemsa stain, and had no history of receiving $H$. pylori eradication therapy. 


\section{Clinicopathological assessment}

Clinicopathological findings such as tumor size, location, macroscopic type, histological type, and depth of invasion were reviewed for gastric carcinomas according to both the Japanese [9] and World Health Organization classifications [10]. The extent and degree of atrophic gastritis was evaluated endoscopically and histologically and classified into six categories according to the Kimura and Takemoto classification system (C-1 to 0-3) [11].

\section{Endoscopic procedures}

Endoscopic examinations were performed by three experienced endoscopists (H.I, T.T, and N.K) using a magnifying endoscope (GIF-H260Z, H290Z, Olympus Medical Systems, Tokyo, Japan). We used structural enhancement levels of A-8 for conventional endoscopy and B-8 for narrow-band imaging with magnifying endoscopy (NBI-ME). NBI-ME for diagnosing EGC was performed using a systematic classification system based on microvascular and microsurface patterns (the "VS classification"). An irregular microvascular pattern and/or an irregular microsurface pattern with clear demarcation lines are the hallmarks of EGC [12]. EGCs were confirmed using histopathological findings of biopsies and ESD samples.

\section{Statistical analysis}

Data were analyzed using JMP ${ }^{\circledR} 15$ statistical software (SAS Institute Inc., Cary, NC, USA). Continuous variables between two groups were evaluated using the Mann-Whitney $U$ test. Categorical variables were evaluated using the chi-squared test. $P$ values $<0.05$ were considered statistically significant.

\section{Results}

\section{Clinicopathological characteristics and endoscopic features of EGCs of the $H$. pyloriEG and $H$. pyloriPG}

Clinicopathological characteristics and endoscopic features of EGCs of the H. pylori-EG and H. pylori-PG are summarized in Table 1. There were 36 EGCs in 32 patients in the H. pylori-EG and 156 EGCs in 140 patients in the H. pylori-PG. The median patient age in the H. pylori-EG (68.1 \pm 7.3 years) was significantly younger than that in the H. pylori-PG $(71.5 \pm 8.7$ years $)(P=0.038)$. The median lesion size in the $H$. pyloriEG $(12.7 \pm 8.2 \mathrm{~mm})$ was significantly smaller than that in the H. pylori-PG $(16.5 \pm 10.8 \mathrm{~mm})(P=0.049)$. EGCs were frequently detected in the upper region of the stomach in six of 36 patients in the H. pylori-EG $(17 \%)$ compared with five of 156 in the H. pylori-PG (3\%; $P=0.002)$. Regarding macroscopic features, elevated lesions were more frequent in 57 of 156 patients in the H. pylori-PG (36\%) compared with six of 36 in the H. pylori-EG (17\%; $P=0.022)$. Depressed lesions were more frequent in 30 of 36 patients in the H. pylori-EG (83\%) compared with 99 of 156 in the H. pylori-PG $(64 \% ; P=0.022)$. Close type-3 in extent of atrophic gastritis according to Kimura and Takemoto classification system [11] was more frequent in the H. pylori-EG (8/36: 22\%) compared with the H. pylori-PG (9/156: 6\%) $(P=0.0002)$. Open type-1 was more frequent in the H. pylori-PG (67/156: 43\%) compared with the H. pylori-EG $(6 / 36: 17 \%)(P=0.0002)$. EGCs 
after $H$. pylori eradication were characterized by features of the small and depressed type, and frequently detected in the upper region of the stomach compared with EGCs in the H. pylori-PG. For histological findings such as histological type, depth of invasion, and lymphatic and venous invasion, there were no differences between the two groups.

\section{Association between characteristics of EGCs and the degree of mucosal atrophy in the $H$. pyloriEG}

Clinicopathological characteristics and endoscopic features of EGCs of the mild atrophic gastric mucosa subgroup and the moderate to severe atrophic gastric mucosa subgroup within the H. pylori-EG are summarized in Table 2. However, six lesions (21\%) were in the mild atrophic mucosa subgroup and 23 lesions (79\%) were in the moderate to severe atrophic mucosa subgroup. Three of six lesions in the mild atrophic subgroup (50\%) had no atrophic mucosa around the EGC. There were no significant differences in age, time interval from eradication of $H$. pylori to detection of EGC, and tumor location, size, macroscopic findings, histological type, or lymphatic and venous invasion between the two subgroups. In the moderate to severe atrophic subgroup, there were more male patients than in the mild atrophic mucosa subgroup $(P=0.02)$. Regarding depth of invasion, submucosal invasive EGC lesions were detected in four of six lesions (67\%) in the mild atrophic mucosa subgroup and in only one of 23 lesions $(4 \%)$ in the severe atrophic mucosa subgroup $(P=0.003)$. Three of four submucosal invasive EGC lesions (75\%) in the mild atrophic subgroup had no atrophic mucosa around the EGC.

\section{Median time interval from eradication of $H$. pylori to detection of EGC}

In the H. pylori-EG, EGCs were detected $1-17$ years (median, 4.6 years) following $H$. pylori eradication. In the mild atrophic gastric mucosa subgroup within the H. pylori-EG, the median interval from eradication therapy to detection of EGC was 5.3 years (1-17 years). In the moderate to severe atrophic gastric mucosa subgroup, the median interval was 4.3 years ( $1-17$ years). Median intervals were not significantly different between the two subgroups (Fig. 2). This showed that there was no significant association between the duration of EGC incidence and the degree of mucosal atrophy.

\section{Submucosal invasive EGC in the H. pyloriEG group}

The clinicopathological characteristics and endoscopic features of five submucosal invasive EGCs in the mild atrophic gastric mucosa subgroup and moderate to severe atrophic mucosa subgroup within the $H$. pylori-EG are summarized in Table 3. Endoscopic features of EGCs in all cases showed a flattened and extended appearance when the entire stomach wall was distended with a high volume of air. Therefore, patients were diagnosed with intramucosal GC because they were negative for the nonextension sign [13]. Three cases (1,3, and 4) had no atrophic mucosa around the EGC. Endoscopic and pathological findings of cases 1 and 4 are shown in Figures 3 and 4, respectively. In case 1, upper gastrointestinal (GI) endoscopy revealed a reddish small elevated lesion in the upper region of the gastric cardia (Fig. 3a). NBIME revealed a regular microsurface pattern and an absent microvascular pattern (Fig. 3b). The tumor was successfully removed en bloc using ESD (Fig. 3c). The resected specimen was evaluated histopathologically and revealed a well-differentiated tubular adenocarcinoma invading the submucosa 
(SM1) (Fig. 3d), with no atrophic mucosa around the EGC (Fig. 3e). In case 4, upper GI endoscopy revealed a reddish small depressed lesion on the upper region of the gastric cardia (Fig. 4a, b). The tumor was successfully removed en bloc using ESD (Fig. 4c). Histopathological examination revealed a welldifferentiated tubular adenocarcinoma invading the submucosa (SM2) and no atrophic mucosa around the EGC (Fig. 4d).

\section{Discussion}

A meta-analysis of clinical studies indicated that gastric cancers occurred even after $\mathrm{H}$. pylori eradication $[5,14,15]$, and therefore it is important to recognize the clinical and histological characteristics of gastric cancers detected after $H$. pylori eradication to make the appropriate diagnosis and treatment. A previous report described 16 gastric cancers detected after $H$. pylori eradication that were characterized by a noncardiac location and $<20 \mathrm{~mm}$ in size with depressed type features [16]. Furthermore, 96 gastric cancers detected after $H$. pylori eradication had endoscopic features of a depressed type and were small compared with H. pylori-positive cancers [5]. Gastric cancers detected after H. pylori eradication were characterized by small size, depressed type, and lower Ki-67 labeling index compared with H. pyloripositive gastric cancers [4]. Likewise, in our study, the H. pylori-EG had more frequent small lesions and depressed types compared with the H. pylori-PG $(P=0.049, P=0.022)$. The current study also showed that the features of EGC after $H$. pylori eradication were characterized as small lesions of the depressed type.

The prevalence of gastric cancers located in the upper region of the stomach was higher in the eradication group compared with the no-eradication group, which suggests that gastric cancer developed in the upper region of the stomach after $H$. pylori eradication [6]. Our study showed the same tendency, that is, EGCs were detected more frequently in the upper region of the stomach in the H. pylori-EG compared with the H. pylori-PG $(P=0.002)$. Hence, it is necessary to perform careful examination of the upper region of the stomach using upper $\mathrm{GI}$ endoscopy after treating a patient with $H$. pylori eradication therapy.

Several studies showed that patients with gastric cancer detected after eradication of $H$. pylori had severe atrophic gastritis $[16,17]$. Atrophy of gastric mucosa in the antrum, angle, and corpus, and intestinal metaplasia in the lesser curvature of the corpus showed significant improvement during the 10-year period after eradication therapy. Therefore, the improvement of gastric atrophy and intestinal metaplasia was associated with a reduction in gastric cancer occurrence [18]. However, the risk of developing a diffuse-type gastric cancer increases over time in patients with mild to moderate gastric atrophy before $H$. pylori eradication [19]. In our study, six of 29 cases (21\%) had no to mild atrophic gastritis around the EGC that occurred after $H$. pylori eradication. Our result strongly suggested that there was a risk of incidence of EGC even in patients with no to mild atrophic gastritis after $H$. pylori eradication, although the risk was lower than in patients with severe atrophic gastritis. 
Recently, several studies reported that there were cases of submucosal invasive EGCs after $H$. pylori eradication. In 162 patients with $H$. pylori eradication or non- $H$. pylori eradication, submucosal invasive EGCs were detected more frequently in the $H$. pylori-eradication group (13/81 patients, $16 \%)$ than in the non- $H$. pylori-eradication group (4/81 patients, $4.9 \% ; P=0.021)$ [20]. The submucosal invasive gastric cancer tended to be more frequent in the H. pyloriEG (18\%) than in the non-H. pylori-EG $(8 \% ; P=0.051)$ [5]. We described four cases of submucosal invasive EGCs with no to mild atrophic gastric mucosa after H. pylori eradication therapy. They were endoscopically diagnosed as intramucosal gastric cancer because of a negative nonextension sign as shown in case presentation [13]. Despite no to mild atrophy after $H$. pylori eradication, it is necessary to follow up using $\mathrm{Gl}$ endoscopy because of the possibility of EGC and the high risk of invasive cancer.

A 9-year prospective study in Japan showed that 20 (1.1\%) gastric cancers were diagnosed in 1,787 patients who underwent $H$. pylori eradication therapy. Of these 20 gastric cancers, $16(80 \%)$ were diagnosed within 4 years after $H$. pylori eradication [16]. Previous studies reported that the median intervals from $H$. pylori eradication therapy to detection of gastric cancers were 2.6 years (range $0.8-11$ years) [4], 4.1 years ( $1-15$ years) [5], and 3 years ( $0.5-15$ years) [6]. In the present study, the median interval was 4.6 years (1-17 years). In the mild atrophic gastric mucosa subgroup of the H. pylori-EG, the median interval was 5.3 years (1-17 years). In the moderate to severe atrophic gastric mucosa subgroup, the median interval was 4.3 years (1-17 years). We suggested that upper $\mathrm{Gl}$ endoscopic examination should be performed in patients in whom $\mathrm{H}$. pylori had been eradicated for at least 6 years, regardless of the degree of atrophic mucosa. However, few cases of EGC were detected 15 or 17 years after H. pylori eradication therapy. Endoscopic surveillance should be continued beyond 10 years after $H$. pylori eradication regardless of the degree of gastric mucosal atrophy [19].

\section{Conclusions}

Our single-center study revealed that EGCs after $\mathrm{H}$. pylori eradication were characterized endoscopically with features of small and depressed lesions and invasive carcinogenesis in the no to mild atrophic mucosa subgroup. Therefore, we should perform careful follow-up with upper GI endoscopic examination after $H$. pylori eradication, with special attention to small and depressed-type gastric cancer for at least 6 years, but not more than 15 or 20 years. This study had several limitations. The study was retrospective and conducted at a single center. Because few reports have evaluated EGCs after $H$. pylori eradication, it will be necessary to perform a prospective study across multiple patient populations and ethnic groups in the future.

\section{Abbreviations}

H. pylori: Helicobacter pylori, EGC: Early gastric cancer, ESD: Endoscopic submucosal dissection 
UBT: ${ }^{13} \mathrm{C}$-Urea breath test, NBI-ME: Narrow-band imaging with magnifying endoscopy

GI: Gastrointestinal

\section{Declarations}

\section{Ethics approval and consent to participate}

This study was conducted in accordance with the ethical standards of the declaration of Helsinki. This study protocol was approved by the Human Ethics Review Committee of Fukuoka University, Japan (IRB number: U20-01-011). Informed consent to participate in this study was obtained verbally from all patients. The procedure for verbal consent was approved by the ethics committee.

\section{Consent for publication}

Not applicable.

\section{Availability of date and materials}

The datasets used and analyzed in the current study are available from the corresponding author on reasonable request.

\section{Competing interests}

The author declare that they have no competing interests.

\section{Funding}

This study received no financial support.

\section{Authors' contributions}

$\mathrm{HI}$ and $\mathrm{FH}$ designed the research. $\mathrm{HI}, \mathrm{TT}, \mathrm{SI}, \mathrm{HM}, \mathrm{HY}, \mathrm{HS}, \mathrm{SM}, \mathrm{YK}, \mathrm{NK}, \mathrm{KA}$, and SF performed the research. $\mathrm{HI}$, TT performed the statistical analysis. HI, HT, and FH wrote the paper. All authors read and approved the final manuscript.

\section{Acknowledgements}

We thank Mark Abramovitz, PhD and Cathel Kerr, BSc, PhD from Edanz Group (https://en-authorservices.edanzgroup.com/ac) for editing a draft of this manuscript.

\section{References}

1. International Agency for Research on Cancer: IARC Monographs on the Evaluation of Carcinogenic Risk to Humans, Vol 61: Schistosomotes, Liver Flukes and Helicobacter pylori. IARC; Lyon: 1994 
2. Uemura N, Mukai T, Okamoto S, et al. Effect of Helicobacter pylori eradication on subsequent development of cancer after endoscopic resection of early gastric cancer. Cancer Epidemiol Biomakers Prev. 1997;6:639-42.

3. Fukase K, Kato M, Kikuchi S, Japan Gast Study Group, et al. Effect of eradication of Helicobacter pylori on incidence of metachronous gastric carcinoma after endoscopic resection of early gastric cancer: an open-label, randomized controlled trial. Lancet. 2008;372:392-7.

4. Yamamoto K, Kato M, Takahashi M, et al. Clinicopathological analysis of early-stage gastric cancers detected after successful eradication of Helicobacter pylori. Helicobacter. 2011;16:210-6.

5. Maehata Y, Nakamura S, Esaki M, et al. Characteristics of Primary and Metachronous Gastric Cancers Discovered after Helicobacter pylori Eradication: A Multicenter Propensity Score Matched Study. Gut and Liver. 2017;11:628-34.

6. Horiguchi N, Tahara T, Kawamura T, et al. Distinct Clinic-Pathological Features of Early Differentiated-Type Gastric Cancers after Helicobacter pylori Eradication. Gastroenterology Research and Practice. 2016; 8230815. doi: 10.1155/2016/8230815

7. Sakitani K, Nishizawa T, Arita M, et al. Early detection of gastric cancer after Helicobacter pylori eradication due to endoscopic surveillance. Helicobacter. 2018;23(4):e12503.

8. Matsuo T, Ito M, Tatsugami M, et al. Gastric cancer development after Helicobacter pylori eradication therapy: a new form of gastric neoplasia. Digestion. 2012;85:61-7.

9. Japanese Gastric Cancer Association. Japanese Classification of Gastric Carcinoma, 3rd English Edition. Gastric Cancer. 2011;14:101-12.

10. Carneiro F, Fukayama M, Grabsch HI, et al. Gastric adenocarcinoma. WHO Classification of tumours of the Digestive System Tumours. 5th ed. Lyon: IRAC Press, 2018: p. 85-95.

11. Kimura K, Takemoto T. An endoscopic recognition of the atrophic border and its significance in chronic gastritis. Endoscopy. 1969;3:87-97.

12. Yao K, Doyama H, Gotoda T, et al. Diagnostic performance and limitations of magnifying narrowband imaging in screening endoscopy of early gastric cancer: a prospective multicenter feasibility study. Gastric Cancer. 2014;17:669-79.

13. Nagahama T, Yao K, Imamura K, et al. Diagnostic performance of conventional endoscopy in the identification of submucosal invasion by early gastric cancer: the "non-extension sign" as a simple diagnostic marker. Gastric Cancer. 2017;20:304-13.

14. Choi J, Kim SG, Yoon H, et al. Eradication of Helicobacter pylori after endoscopic resection of gastric tumors does not reduce incidence of metachronous gastric carcinoma. Clin Gastroenterol Hepatol. 2014;12:793-800.

15. Yoon SB, Park JM, Lim CH, Cho YK, Choi MG. Effect of Helicobacter pylori eradication on metachronous gastric cancer after endoscopic resection of gastric tumors: a meta-analysis. Helicobacter. 2014;19:243-8.

16. Kamada T, Hata J, Sugiu K, et al. Clinical features of gastric cancer discovered after successful eradication of Helicobacter pylori: results from a 9-year prospective follow-up study in Japan. 
Aliment Pharmacol Ther. 2005;21:1121-6.

17. Take S, Mizuno M, Ishiki K, Nagahara Y, Yoshida T, Yokota K, et al. Baseline gastric mucosal atrophy is a risk factor associated with the development of gastric cancer after Helicobacter pylori eradication therapy in patients with peptic ulcer diseases. J Gastroenterol. 2007;42 Suppl 17:21-7.

18. Kodama M, Murakami K, Okimoto T, et al. Ten-year prospective follow-up of histological changes at five points on the gastric mucosa as recommended by the updated Sydney system after Helicobacter pylori eradication. J Gastroenterol. 2012;47:394-403.

19. Take S, Mizuno M, Ishiki K, et al. Risk of gastric cancer in the second decade of follow-up after Helicobacter pylori eradication. J Gastroenterol. 2020;55:281-8.

20. 20. Hata K, Ito M, Kotachi T, et al. Gastric Cancer with Submucosal Invasion after Successful Helicobacter pylori Eradication: A Propensity Score-Matched Analysis of Patients with Annual Patient Endoscopic Survey. Digestion. 2019;99(1):59-65.

\section{Tables}

Due to technical limitations, Tables 1-3 are provided in the Supplementary Files section.

\section{Figures}




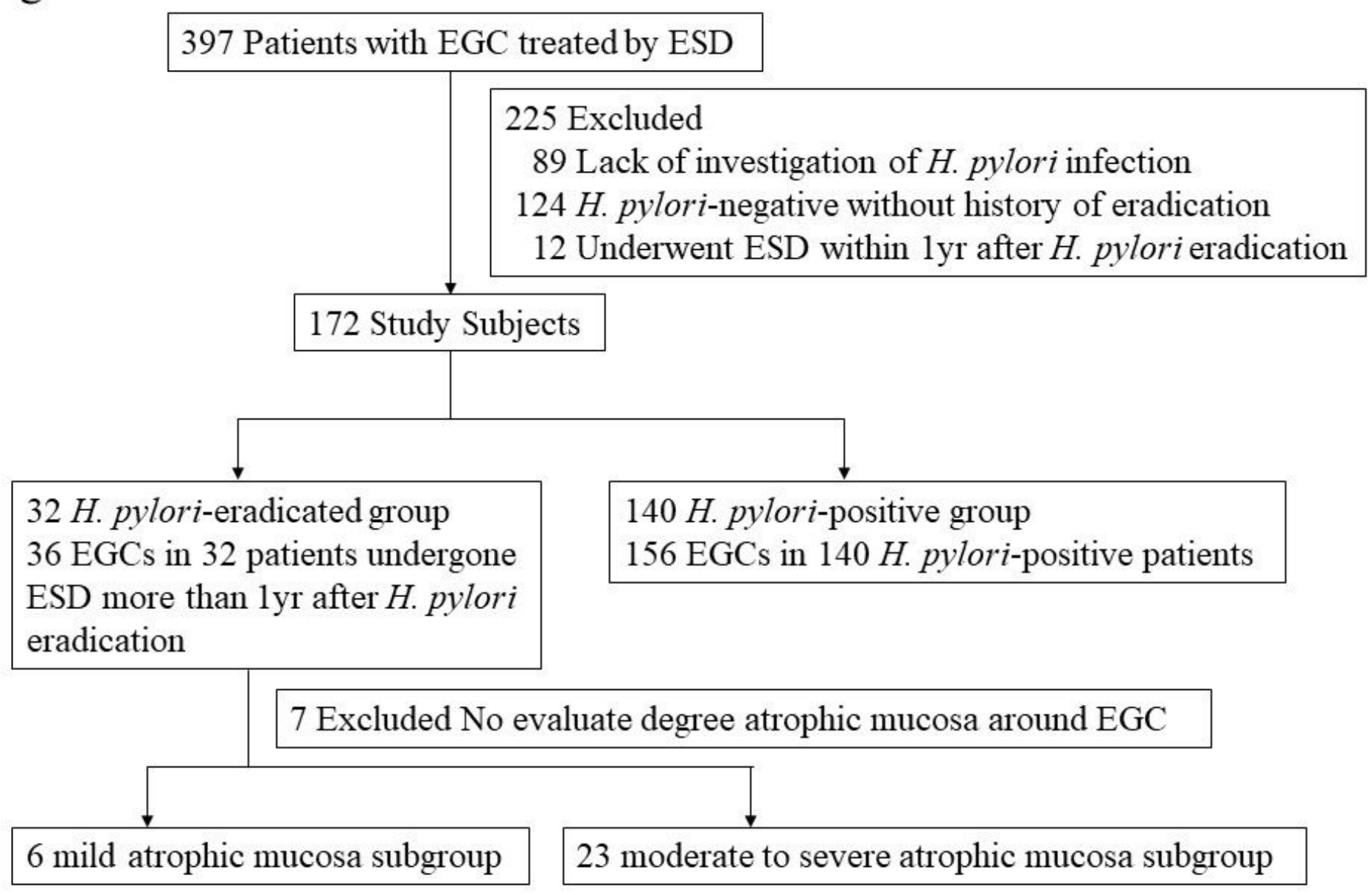

EGC: early gastric cancer, ESD: endoscopic submucosal dissection, H. pylori: Helicobacter pylori

Figure 1

Flow diagram of the study subjects. 
Figure 2

$$
P=0.8487
$$

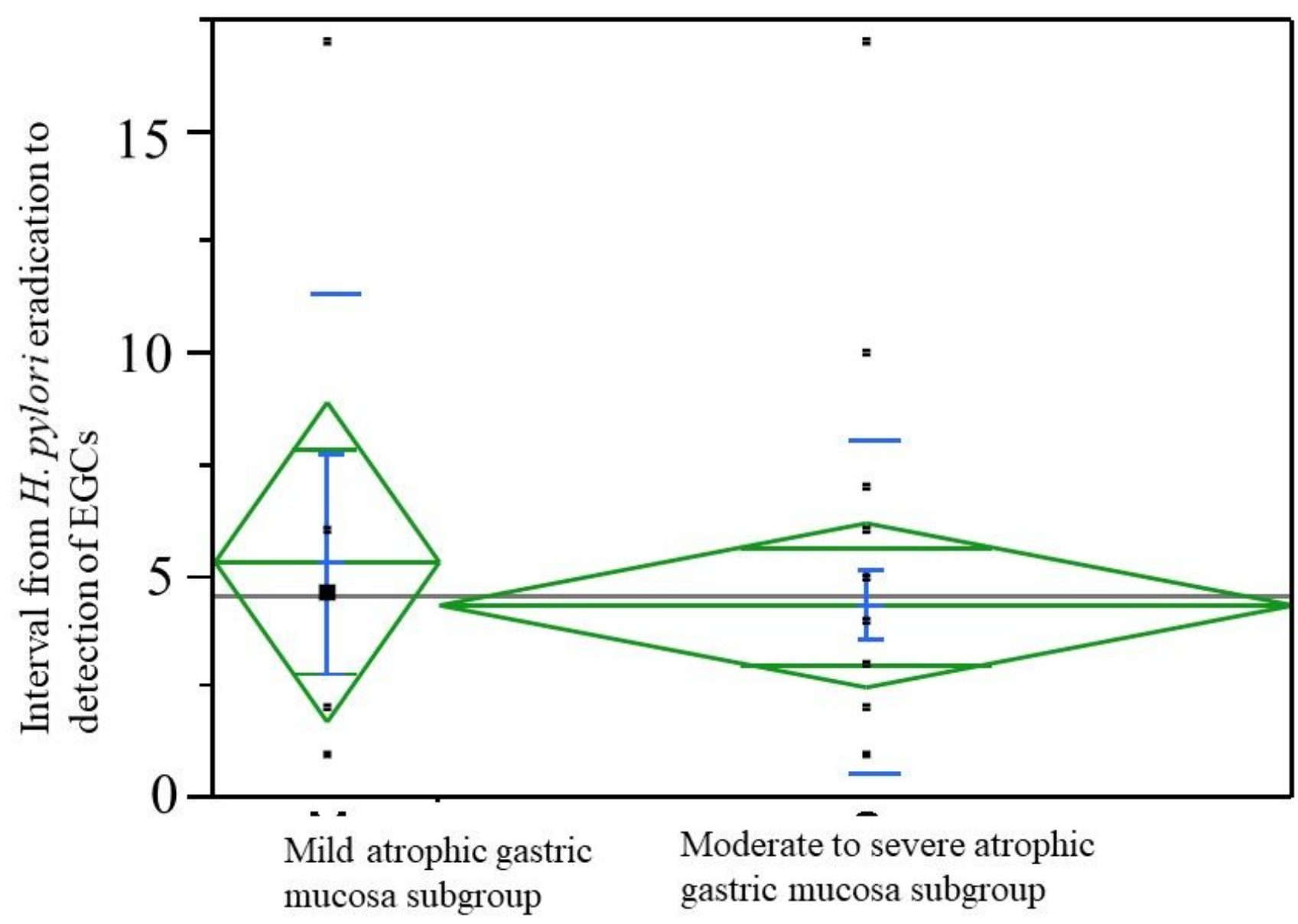

H. pylori: Helicobacter pylori, EGC: early gastric cancer

\section{Figure 2}

Median time interval from eradication of $\mathrm{H}$. pylori to detection of EGC. In the mild atrophic gastric mucosa subgroup in the H. pylori-EG, the median interval from eradication therapy to detection of gastric cancer was 5.3 years (1-17 years). In the moderate to severe atrophic gastric mucosa subgroup, the median interval was 4.3 years (1-17 years). Median intervals were not significantly different between the two subgroups. 


\section{Figure 3}
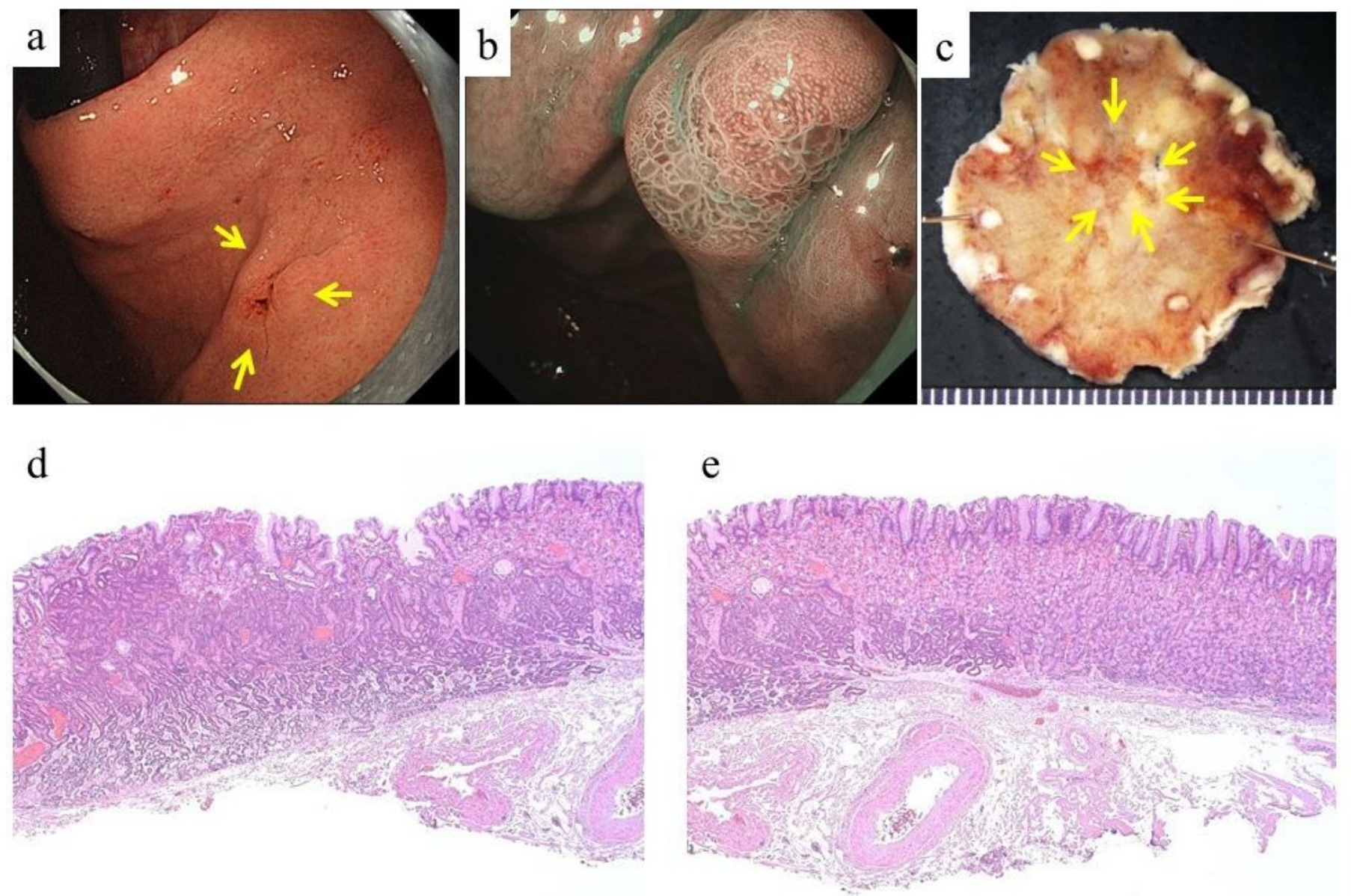

\section{Figure 3}

Case 1 of submucosal invasive EGC in the mild atrophic gastric mucosa subgroup. a: Findings of upper gastrointestinal endoscopy show a reddish small elevated lesion (arrows) of approximately $5 \mathrm{~mm}$ in size in the upper region of the gastric cardia. b: Findings of magnifying endoscopy with narrow band imaging show a regular microsurface pattern and an absent microvascular pattern. c: Macroscopic findings of the resected gastric specimen using ESD show a locally elevated lesion (arrows) of $5 \times 5 \mathrm{~mm}$ in diameter. d: Histopathological findings of the resected gastric specimen using ESD show a well-differentiated tubular adenocarcinoma invading the submucosa (hematoxylin-eosin stain). e: Histopathological findings of the resected gastric specimen using ESD show none atrophic mucosa around early gastric cancer (hematoxylin-eosin stain). 
Figure 4
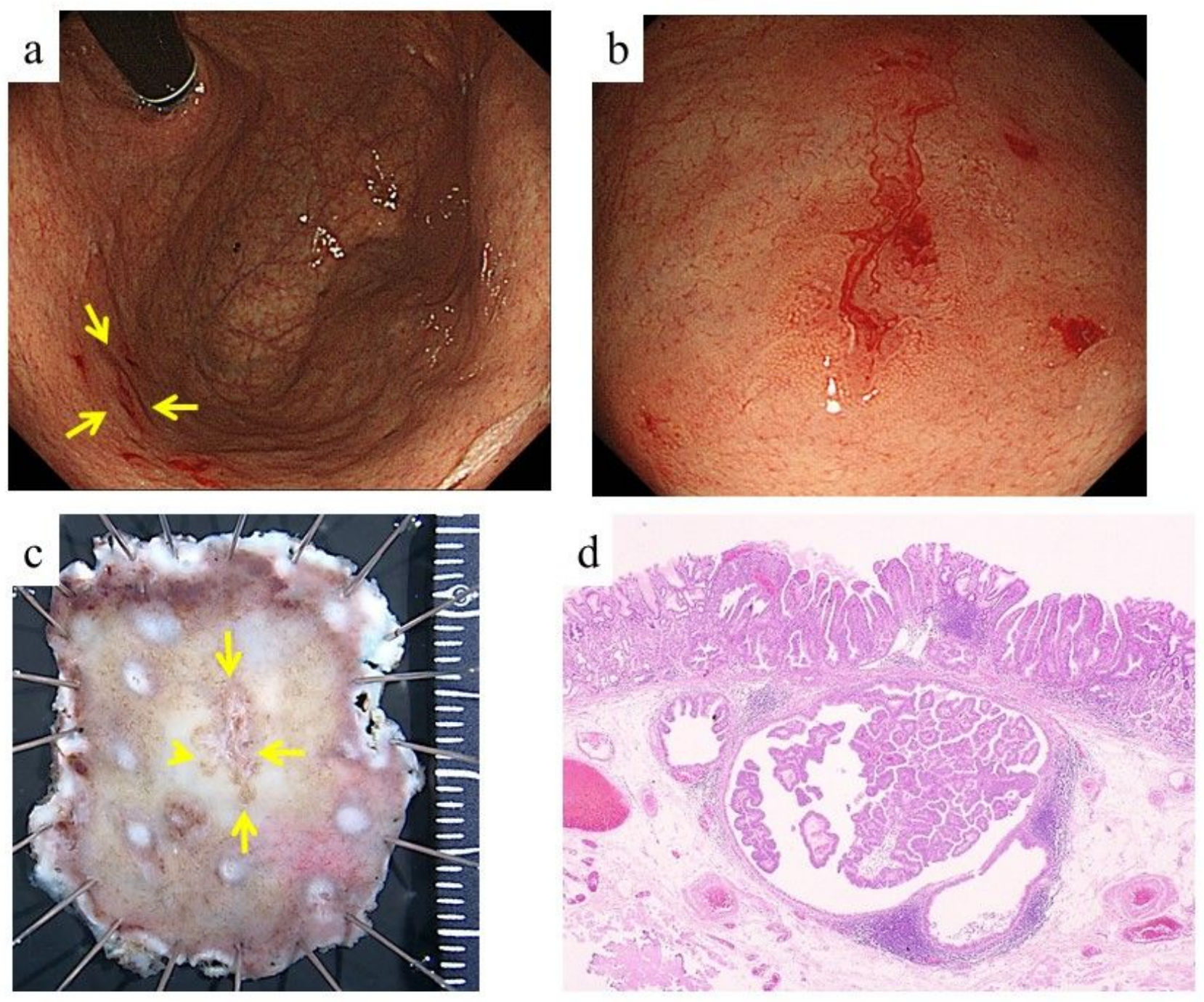

\section{Figure 4}

Case 4 of submucosal invasive gastric cancer in the mild atrophic gastric mucosa subgroup. a: Findings of upper gastrointestinal endoscopy show a small depressed lesion (arrows) of approximately $8 \mathrm{~mm}$ in size in the upper region of the gastric cardia. b: Findings of upper gastrointestinal endoscopy show a reddish small depressed lesion with occult hemorrhage. c: Macroscopic findings of the resected gastric specimen using ESD show a locally depressed lesion (arrows) of $8 \times 4 \mathrm{~mm}$ in diameter. d:

Histopathological findings of the resected gastric specimen using ESD show a well-differentiated tubular adenocarcinoma invading the submucosa (hematoxylin-eosin stain).

\section{Supplementary Files}

This is a list of supplementary files associated with this preprint. Click to download.

- Table.xlsx 